\title{
Spontaneous Chiral Symmetry Breaking in a Random, Driven Chemical System
}

\author{
William D. Piñeros ${ }^{1}$ and Tsvi Tlusty ${ }^{1,2,3, *}$ \\ ${ }^{1}$ Center for Soft and Living Matter, Institute for Basic Science (IBS), Ulsan 44919, Korea \\ ${ }^{2}$ Department of Physics, Ulsan National Institute of Science and Technology (UNIST), Ulsan 44919, Korea \\ ${ }^{3}$ Department of Chemistry, Ulsan National Institute of Science and Technology (UNIST), Ulsan 44919, Korea
}

(Dated: August 9, 2021)

\begin{abstract}
Living systems have evolved to efficiently consume available energy sources using an elaborate circuitry of chemical reactions, which are puzzlingly restricted to specific chiral configurations. While autocatalysis is known to induce such chiral symmetry breaking, whether this might also arise in a more general class of non-autocatalytic chemical networks - by mere virtue of energy source exploitation - is a sensible yet underappreciated possibility. In this work, we examine this question within a model of randomly-generated complex chemical networks and show that chiral symmetry breaking may occur spontaneously and generically by harnessing energy sources from external environmental drives. Key to this transition are intrinsic fluctuations of achiral-to-chiral reactions and tight matching of system configurations to the environmental drive which, together, amplify and sustain diverged enantiomer distributions. The results thus demonstrate a generic mechanism in which energetic drives may give rise to homochirality in an otherwise totally symmetrical environment.
\end{abstract}

\section{INTRODUCTION}

A hallmark of living systems is their robust and intricate self-organization towards the exploitation of energy sources available in the environment. This is readily observed in metabolic networks where chemical reactions are carefully orchestrated to transduce external energy sources into molecular energy currency like ATP. Intriguingly, these biochemical reactions take place with nearexclusive preference for specific chiral configurations over their racemic equivalents, a property termed homochirality $[1,2]$. While the origins of homochirality remain an open question, most mechanisms propose the existence of pre-determinate environmental biases, such as templating-surfaces [3, 4], polarized light [5], or more generally rely on autocatalytic processes to amplify underlying chiral imbalances [6-9]. One such classic example is the Frank model in which homochirality emerges spontaneously under the assumption of autocatalytic production of a chiral molecule and cross-inhibition with its enantiomer [10]. While mutual enantiomer antagonism was thought critical for the model, recent studies by Jafarpour et al. showed that stochastic noise in the formation of chiral molecules sufficed to induce the symmetry breaking [11, 12]. Additionally, a comprehensive study by Laurent et al [13] generalized Frank's single reaction model, and demonstrated that chiral symmetry breaking occurred in large, random reaction systems, further corroborating the generality of the result.

On the other hand, whether purely energetic processes, and without built-in autocatalytic events, could instead mediate chiral symmetry breaking remains an underappreciated but important consideration. For instance, dipole-like interactions lead to well-known symmetrybreaking transitions, and were applied analogously to

\footnotetext{
* tsvitlusty@gmail.com
}

explain induced chiral bias in self-interacting helical polymers, self-assembling nano-crystals and even selfpropelled achiral particles [14-16]. Similarly, an energetic picture is consistent with polymerization models where activation of enantiomer monomers may lead to homochiral states through epimerization cycles $[17,18]$. Indeed, energetic processes bear natural relevance to the origin of homochirality, as intense chemical and energetic environments with steep non-equilibrium gradients are thought to have conditioned the rise of self-adaptive chemical processes conjectured to predate modern living systems [19]. Thus, whether chiral symmetry breaking could also arise spontaneously in a random chemical system out of exploitation from an environmental energy flux seems like a natural yet unaddressed question.

Recently, an example of an energy-driven chemical system was introduced by Horowitz and England and shown to generate highly-dissipating states as a result of continual fuel harvesting from environmental sources [20]. The emergence of these states was attributed to strong matching between system configurations and environmental forcing such that the system becomes more responsive to the drive and thereby increase its energy source exploitation. Similar results have been demonstrated in other driven and self-configurable theoretical and experimental systems [21-23]. These findings then motivate us to ask here whether a similar chiral chemical system could break symmetry via such strong-matched environmental driving. Thus, if such dissipative self-arrangement is sufficiently strong and persistent, then a spontaneous chiral symmetry breaking may take place out of amplification of symmetric but inherently noisy conditions. Such result would therefore be entirely independent of a priori autocatalytic mechanisms, and instead arise exclusively out of configurational arrangements conditioned on the exploitation of the external drive.

In this work we explore this idea and study a complex, random chiral chemical system subject to configurationally-dependent environmental forces. In 
particular, the system generates fluctuations of chiral species via random achiral-to-chiral reactions, and the resulting stochastic dynamics is solved in otherwise totally symmetric conditions. Remarkably, we find that for generic but relatively rare instances of our model, chiral symmetry is spontaneously broken, and the transition is entirely dependent on continuous dissipation from the external drives. As we elaborate below, this event is tied sharply to the difference in work harnessed along the dynamical trajectory of a chiral-breaking system relative to its symmetric counterpart. Chiral symmetry breaking is therefore predominately observed at high-dissipation regimes. Altogether, this finding demonstrates a first instance where chiral symmetry breaking takes place by virtue of a self-configurable system adapting to an energy source.

\section{A Complex Chiral Chemical System}

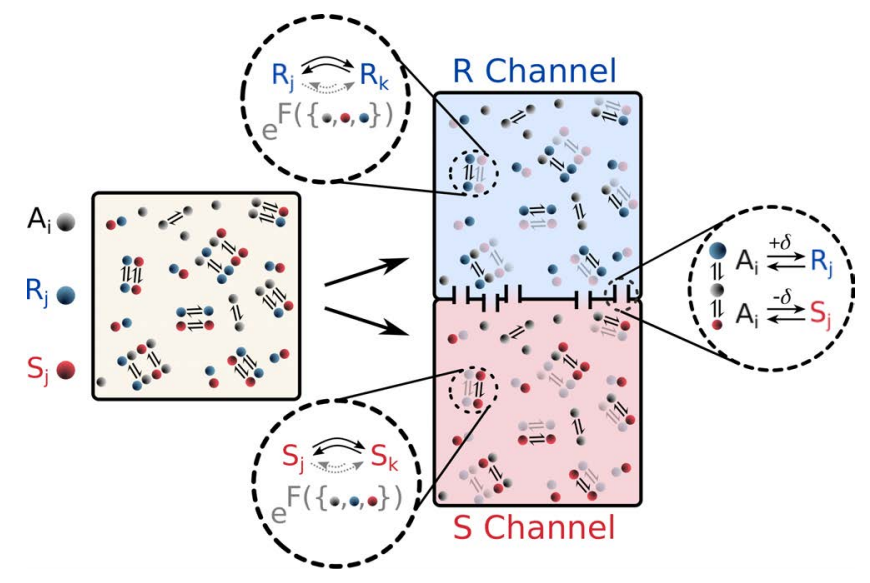

FIG. 1. A. Reaction schematic in a solution of achiral elements $A_{i}$ (gray) and chiral elements $R_{j}\left(S_{j}\right)$, where $R_{j}$ (blue) is the chiral counterpart of $S_{j}$ (red). Under total enantioselectivity, the system (yellow box on left) can be decomposed into quasi-independent but mirrored $R$ (red) and $S$ (blue) configuration channels. Dashed insets show a sample equilibrium chiral reaction between elements $R_{j}\left(S_{j}\right)$ and $R_{k}\left(S_{k}\right)$. A fraction of these reactions is randomly doubled and driven by environmental forces $F^{\gamma}$ which depend on a complex expression of system elements $\left\{A_{i}, R_{j}, S_{k}\right\}$. Force expressions are identical but mirrored (i.e. $R_{j} \rightarrow S_{j}$ and vice-versa) to maintain channel symmetry. The two channels are connected by "canals" (rectangular passages) which allow mass exchange between channels via achiral-to-chiral reactions. This exchange is symmetric but may vary by a random fraction $\delta$ as a result of environmental fluctuations.

We construct the chemical system using a framework recently introduced in [20], where achiral species $\left\{A_{i}\right\}$ react under the influence of external environmental forces. To explore the possibility that coupling to strong environmental drives may induce spontaneous breaking of chiral symmetry, we introduce here additional sets of chiral species $\left\{R_{j}\right\}$ and their corresponding enantiomers $\left\{S_{j}\right\}$.
Specifically, we consider a dilute solution of chiral and achiral elements at equilibrium in a container of constant volume and temperature as shown in Figure 1 left. Species are randomly wired in uni- or bi-molecular reactions, some of which may be randomly catalyzed by another independent species. Enantiomers are assumed to be mutually inert, and cross-reaction between chiral species to be perfectly enantioselective. As a result, any chiral species $i$ can be arbitrarily captured by independent $R_{i}$ and $S_{i}$ sets since otherwise enantioselectivity with respect to species $j$ would break with a trivial swap of $R / S$ element label. This is illustrated in Figure 1 where the total system may be viewed as consisting of two separate $R / S$ "channels" with only the achiral $A_{i}$ elements held in common. Achiral elements then play the critical role connecting $R / S$ channels as mass exchange "canals" through achiral-chiral reactions of the form $A_{i} \rightleftharpoons R_{j}\left(S_{j}\right)$ (Figure 1). This exchange process is symmetric on average, but subject to random fluctuations $\pm \delta$ induced by the environment which manifest as noise in the chiral species dynamics. This small, inherent stochasticity of the system links the quasi-independent channels, thereby allowing for the possibility of a diverging dynamics via strong environmental coupling that would constitute a chirality-breaking event.

Environmental drives are prescribed here through a set of forces $F=\left\{F^{\gamma}\right\}$ that depend on system configurations and induce kinetic tilting of the underlying chemical network. This is achieved by duplicating a random set of existing equilibrium reactions $\alpha$ and favoring (inhibiting) the forward (backward) rate constants by an exponential factor of the applied force, $k_{\alpha}^{\prime \pm}=k_{\alpha}^{ \pm} e^{ \pm \beta F^{\gamma} / 2}$, where $k_{\alpha}^{ \pm}$are equilibrium constants and $\beta=1 / k_{\mathrm{B}} T$ is the usual inverse temperature and Boltzmann constant. The force itself is a function that may depend on any subset of concentrations, $F^{\gamma}\left(\left\{A_{i}, R_{j}, S_{k}\right\}\right)$. The functions are constructed based on a model known to generate frustrated pair dynamics (see Methods). Due to the chiral composition of each channel, forces are functionally identical but mirrored, i.e., they are related through a corresponding $R / S$ label swap in the expression as $\left\{A_{i}, R_{k}, S_{j}\right\} \longleftrightarrow\left\{A_{i}, S_{k}, R_{j}\right\}$. This ensures the system is kept symmetric, and allows us to deduce the emergence of any diverging dynamics as the result of chiral breaking and not a prescribed environmental bias.

\section{RESULTS}

Highly-dissipating systems may spontaneously break chiral symmetry

Having defined the chiral chemical model, we now explore our main question: may a totally symmetric system spontaneously break chirality by matching to an external environmental drive? To this end, we consider a system of $N_{c}=12$ chiral pairs and $N_{a}=2$ achiral elements for a total of $N=26$ elements. The stochastic dynam- 

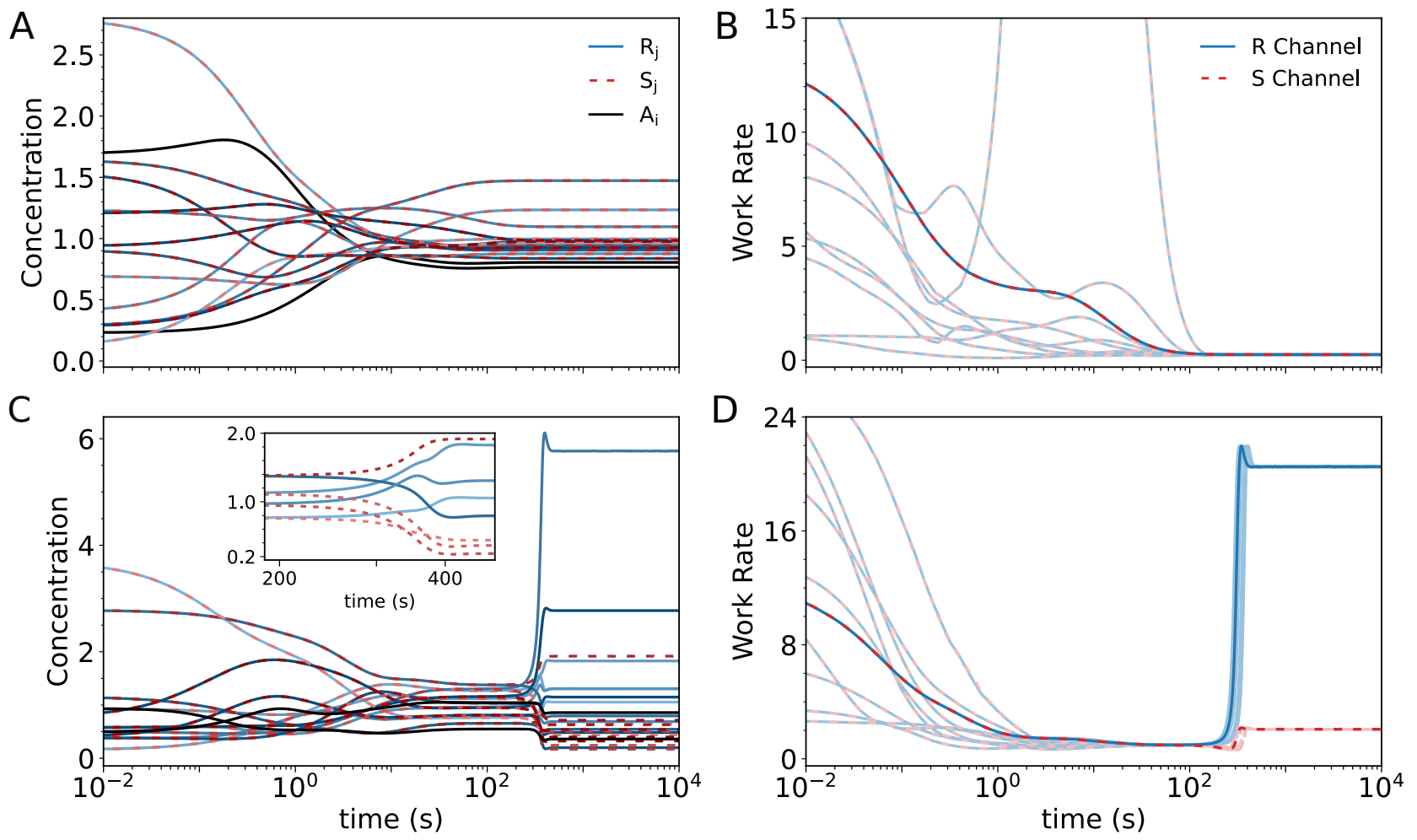

FIG. 2. Concentrations as a function of time for a typical chiral-conserving system (A) and a chiral-breaking model (C). Inset shows close-up trajectories of four representative chiral pairs shortly after chiral breaking, here seen as bifurcations from left to right. Work rate plots as a function of time for the same chiral-conserving (B) and chiral-breaking models (D) for 10 random initial conditions. Here, only $R$ dominant results - defined as the channel achieving the maximum work rate in a given run-are selected and shown for clarity. Channel dominance is random and equally distributed on average $(R, S=50 \pm 2 \%$, in $4 \times 50$ run batches).

ics of the system are then simulated, starting from random but symmetric initial conditions $\left(i . e ., R_{i}(0)=S_{i}(0)\right.$, where $R / S(t)$ represent element concentrations as a function of time). Typically, such runs are racemic and replicate the results of [20], with the majority yielding fixed-point, moderately-driven steady-states and a few displaying highly-dissipating states. Similar results hold for other model parameters (see Methods).

In figure $2 \mathrm{~A}$, we show an example of one such racemic system for a moderately-driven model, where chiral symmetry is clearly conserved across the entire run and elements settle at near-equilibrium concentrations as a result of the moderate-drive. To quantify the strength of this driving process, we compute the rate at which work is performed by the drives to power the system, here defined as $\dot{W}=\sum_{l} F_{l}^{\gamma}\left|J_{l}\right|$, where the net rate of formed molecules or reaction currents, $J_{l}$, is defined as $J_{l}=k_{l}^{+} X_{j}-k_{l}^{-} X_{i}$. Thus, as seen in figure $2 \mathrm{~B}$, the overall drive performance is low at the steady-state, but applies symmetrically on both chiral channels as per a racemic state.

In contrast, and very remarkably, for some exceptional instances of our model, the initial symmetrical trajectories of the system can suddenly split and diverge as a result of spontaneous chiral symmetry breaking. This is seen clearly in figure $2 \mathrm{C}$ for a representative chiralbreaking model, where the concentration profiles of chiral elements bifurcate rapidly at around 200 s, and demonstrate strong asymmetric divergence. Chiral bifurcation is equally apparent in the plots of system work-rates (figure 2D) which in this model manifests as a dominant, highly-driven channel over its weaker chiral counterpart. In addition, as expected from symmetry, the outcome of channel dominance does not depend on its chiral label, but is random and equi-probable upon reruns. Furthermore, the time of symmetry-breaking onset is variable and depends on noise history. These results hold generically across all obtained chiral-breaking models, suggesting that symmetry breaking is not the result of built-in biases, but originate from the dynamical matching between the reactive system and the environmental drives.

\section{Large energy differences induce chiral biases}

Next, we explore this dynamical picture systematically. In particular, we examine how the emergence of chirality-breaking events - which manifest as sudden, but reinforcing divergent dynamics - is linked to a corresponding sudden energy absorption by the system from 

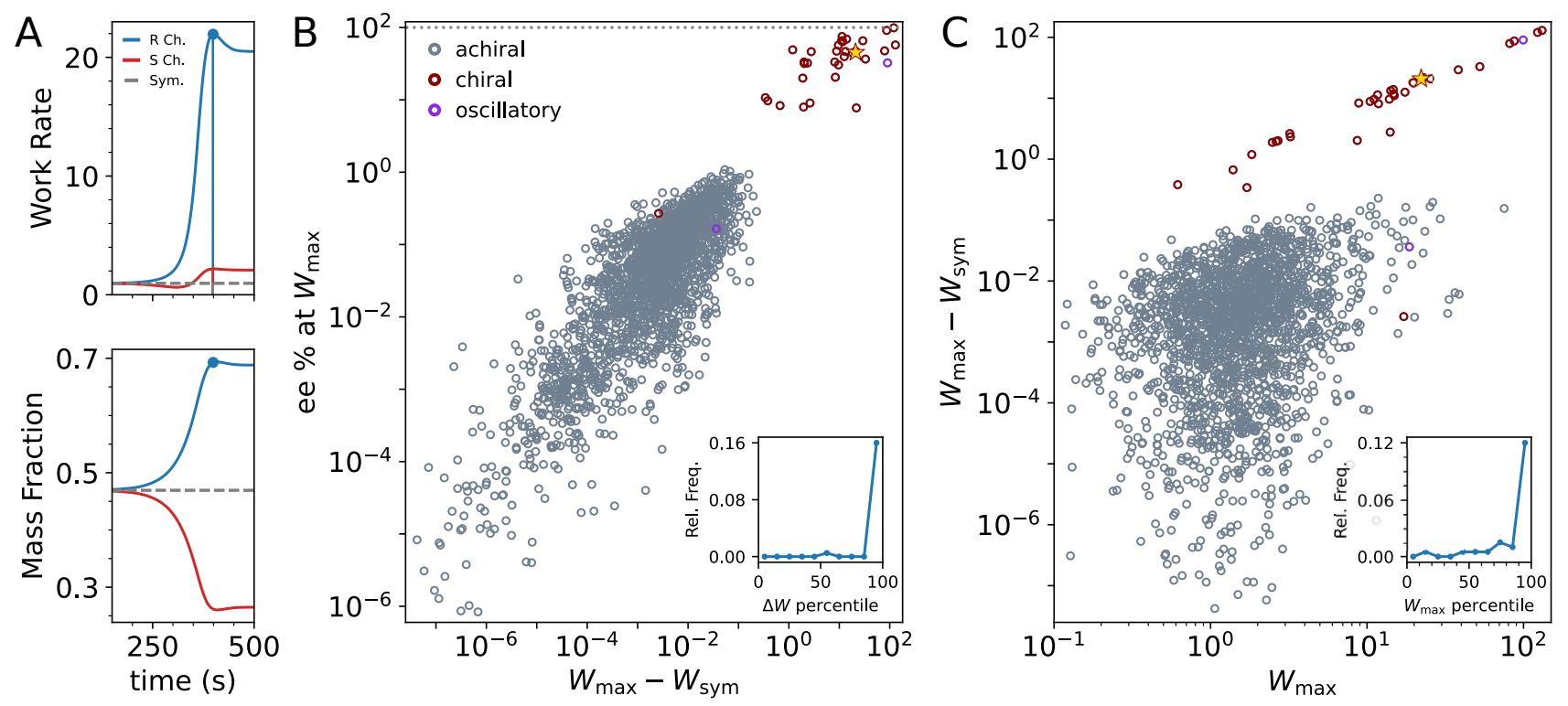

FIG. 3. A. The work rate (top) and mass channel fractions (bottom) for the chiral breaking system in figure 2 and its corresponding symmetrical case (note linear time axis). Lines under curves denote work done by the drives over the span of one second at maximum channel work rate $W_{\max }$ (blue marker) B. The enantiomeric excess ee percent at $W_{\max }$ vs. the work difference, $\Delta W=W_{\max }-W_{\text {sym }}$, between the symmetric and chiral breaking trajectories for 2,000 random, independent models (points below $\sim 10^{-8}$ omitted for clarity). Slate dots denote chiral-conserving models and red dots chiral-breaking models. Yellow star denotes the system drawn in A. Purple dots denote a very rare class of solutions displaying large ee oscillations as a function of time but with average $e e=0$ over a complete cycle. Inset: relative population frequency of chiral-breaking models as a function of the work difference percentile. C. $\Delta W$ vs. $W_{\max }$ for the same data set as B. Inset: the relative population frequency of chiral-breaking results as a function of the maximum work percentile.

the drive. Physically, this could be thought as an energy injection driving a phase transition. Hence, we identify chiral-breaking models and compare them to perfectly symmetrical counterparts where enantiomers are strictly equal at all times, i.e., $S_{i}(t)=R_{i}(t)$. Using the same starting conditions, we then re-ran both system versions and computed their difference in maximum harnessed-work over an equal-time span after bifurcation, $\Delta W=W_{\max }-W_{\text {sym }}$, where $W_{\max }$ and $W_{\text {sym }}$ represent the work for the actual and symmetric case respectively. Furthermore, if chiral symmetry breaking depends on $\Delta W$ then it should also correlate with the degree of divergence, here computed as the channel enantiomeric excess, $e e=\left|m_{R}-m_{S}\right| /\left(m_{R}+m_{S}\right)$, where $m_{R(S)}$ is the total mass fraction of chiral elements in each channel. As an example, we thus consider again the chiral model in figure $2 \mathrm{~A}$, and plot the work rates, and mass fractions $m_{R(S)}$ for the symmetric and chiral-system in figure 3. Clearly, mass bifurcation correlates strongly with elevated work-rate dissipation, and is consistent with an energy-determined event not present in the symmetrical dynamics.

The comparison is made systematic by plotting $\Delta W$ against $e e$ for 2,000 independent models for chiralbreaking and racemic models as shown in figure 3B. Indeed, we find that the values of $e e$ and $\Delta W$ follow a strong positive trend and cluster into distinct sets of chiral-breaking and racemic models, demonstrating that chiral-breaking events are generally followed by large $\Delta W$ differences. This is made evident by computing the frequency of symmetry breaking systems relative to population as a function of the percentile ranking of $\Delta W$ as shown in inset. Clearly, symmetry-breaking events are sharply over-represented at exceptionally large values of the harnessed-work difference. Very rarely, $\Delta W$ may also be small and still induce chiral symmetry breaking, or generate oscillating solutions with large instant $e e$ values but zero average (see figure S1). As we elaborate later, these rare situations simply highlight that finite $\Delta W$ differences must also result in the strong matching of the system with the drive so as to generate and sustain a diverged dynamics.

It is also instructive to examine how $\Delta W$ relates to the overall magnitude of driving extracted by the system as represented by $W_{\max }$ (figure $3 \mathrm{C}$ ). Is harnessing a larger overall output from the drive more likely to result in large $\Delta W$ values, for instance? We find that large $\Delta W$ and $W_{\max }$ are indeed strongly correlated in the chiral-breaking models and are almost equal by virtue of $W_{\text {max }} \gg W_{\text {sym }}$, except for the few outliers at cluster periphery. Likewise, the relative frequency of chiralbreaking models is strongly peaked at high percentiles of $W_{\max }$, showing together, that strong environmental driving is generally precursive to symmetry breaking. We emphasize these results hold generically for various instances of the model (figure S2). 

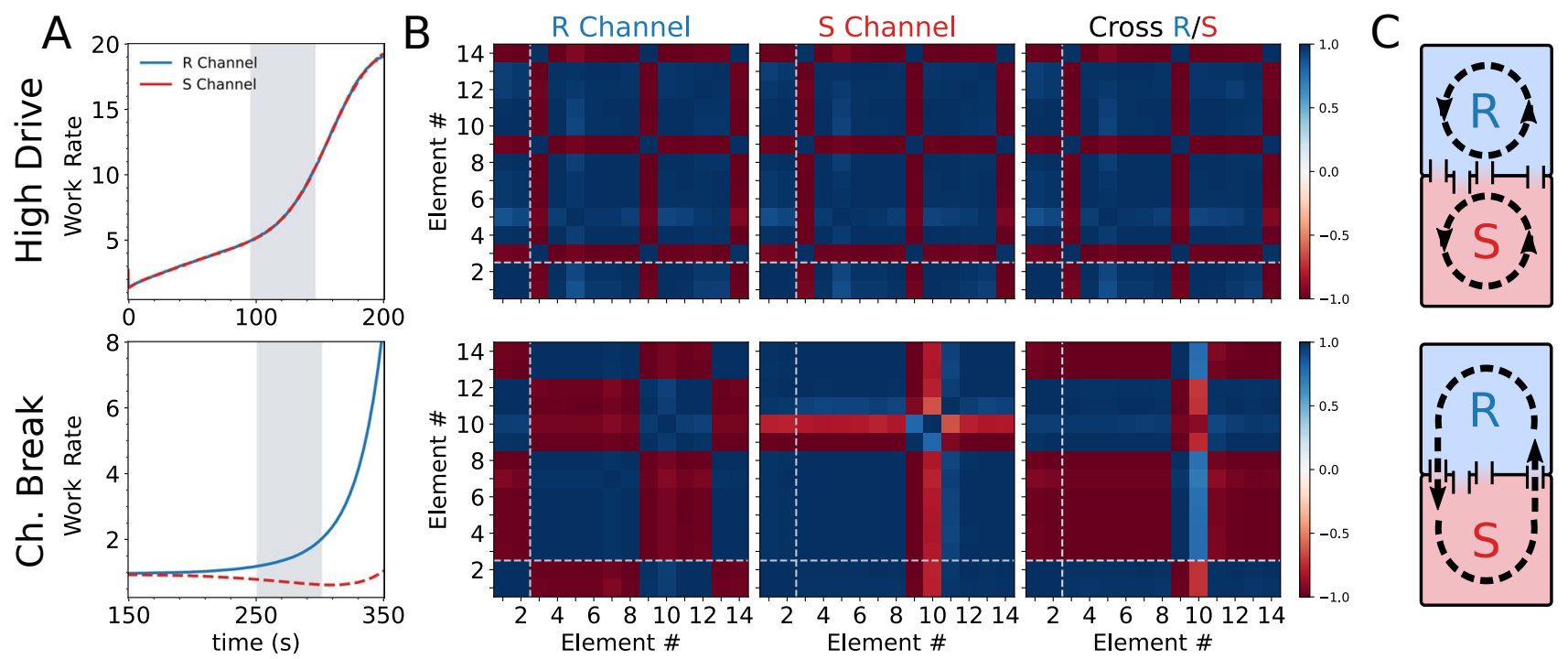

FIG. 4. A. Work rates for a high drive system (top) and the chiral breaking model of figure 2 (bottom). B. Concentration correlation matrices between all achiral $(1-2)$ and chiral $(3-14)$ elements of each corresponding $R$ or $S$ channel over a period of $50 \mathrm{~s}$ (shaded areas in A). Dashed light colored lines denote achiral-chiral element correlation boundaries. Correlation time ranges are chosen to be at comperative time-scales of the bifurcation horizon for the chiral-breaking system and the onset of strong-drive in the chiral-conserving model. Third matrix column shows the cross-channel concentration correlation where rows(columns) correspond to the $R(S)$ channel elements. Color bar denotes maximum and minimum values for all matrices, with $+(-) 1$ indicating perfect positive(negative) correlation. C. Schematic of channel correlations symbolized by counterclockwise cycles indicating a strong matching of the system to the environmental drive. For the chiral-conserving case these are separate and equal (top), but system-wide for the chiral-breaking model (bottom).

Systemwide correlations are required to maintain chiral states

We now clarify how the character of the system-todrive coupling determines, and sustains different kinds of far-from-equilibrium steady-states. In particular, high dissipating achiral and chiral states are both possible, and must clearly differ in mechanism. To examine this issue, we evaluate the correlations coefficients among element concentrations for a representative racemic highdissipation case, and the chiral-breaking system of figure 2 within and across channels. The correlation is calculated over a time window in which each system is near the onset of strong-driving and for comparative timescales as shown in figure 2. Indeed, while both systems display strong intra-channel correlations, the racemic system only replicates correlation patterns across channels as consistent with separate yet identical, mirrored system behavior. On the other hand, channel correlations are clearly distinct for the chiral symmetry breaking case. In particular, elements are strongly anticorrelated across channels, indicative of the sustained, but asymmetric system-wide mass-transfer taking place. This picture then explains why chiral-breaking states are possible even for relatively small values of $\Delta W$ provided that i) strong correlations with the drive take place system-wide, and ii) they are sustained beyond symmetry breaking onset.

\section{DISCUSSION}
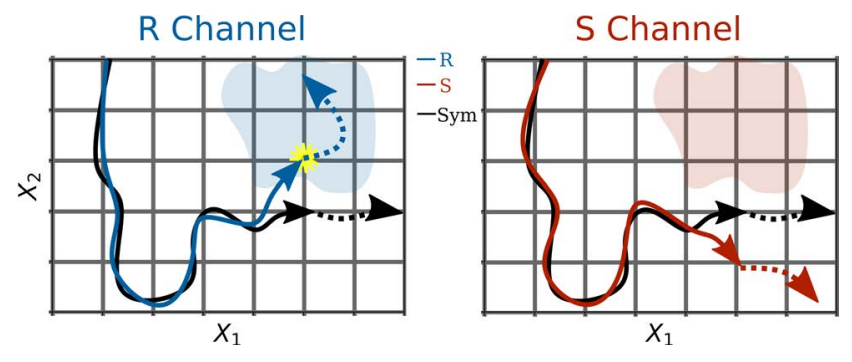

FIG. 5. A generalized trajectory perspective of a chiralitybreaking event for the $R$ (left) and $S$ (right) system channels. Here, $X_{1}$ and $X_{2}$ represent arbitrary state-space coordinates and shaded areas indicate special state-space configurations finely-matched to strong driving from the environment. Yellow asterisk in left plot indicates the point where a random fluctuation in the $R$ trajectory (blue) intersects with the special high driving region and triggers a system bifurcation (seen as diverging dashed lines in both $R$ and $S$ channels). This intersection event is random and equally likely for both channels upon system restart.

The abrupt change in system dynamics at the chirality transition motivates we look at the system-drive evolution from a dynamic-trajectory perspective. In particular, all possible system configurations reside in an $N$-dimensional space of elements' concentrations $X=$ $\left\{A_{i}, R_{j}, S_{k}\right\}$. While such a space is admittedly large, one 
may still envision a projected space of effective system coordinates $X_{i}$ which capture the dominant dynamical modes of the system. Hence, a dynamical trajectory in this space would evolve in $X_{i}$ until reaching a final steady state value.

The complex chiral chemical system of consideration could therefore be pictured as two trajectories representing the $R$ and $S$ channels traversing a parallel but identical state-space as a result of some external drive (figure 5). Upon introduction of environmental noise in the chiral elements, trajectories may make small deviations from, but still generally follow, the symmetric trajectory. However, for a special class of models, trajectories might graze by strong-driving regions which, if crossed, will trigger a sudden divergence in channel dynamics. Because trajectory fluctuations are random, and generally anti-correlated, only one such channel trajectory will reach this region resulting in the spontaneous breaking of chiral symmetry. Of course, given the inherent symmetry of the underlying noise, each trajectory has equal crossing chance, so that the final chiral configuration is arbitrary and equi-probable upon restart.

This phase space picture then implies that achieving a higher-dissipating state relative to symmetric conditions can induce chiral symmetry breaking and is consistent with the above analysis of harnessed-work. Further these regions are only accessible by asymmetric states (through fluctuations) as they exist, by definition, outside of the symmetric state space. As such, sustained symmetry-breaking is entirely dependent on the energy flux from the drives, as otherwise trajectories would inevitably follow the symmetric dynamics. We remark the present results stand in contrast to conventional chiralsymmetry breaking models that require pre-determinate initial biases or built-in autocatalytic mechanisms. Here, instead, chiral symmetry breaking may arise entirely as a result of realizing special matching conditions with the drive, leading to sustained, and enhanced energy proliferation. These system-drive matchings do manifest as system-wide correlations, likely in the form of network cycles, but are time-dependent, configurationally variable and dense. This implies that the extent of chiral symmetry breaking depends as much on the strength of harnessed work as it does on the degree of matching, as evident by the distribution of channel $e e$ values observed for chiral breaking systems in fig 3 . Thus, unlike autocatalytic models with fixed homochiral points, these systems can span an entire spectrum of asymmetrical possibilities and suggest an additional mechanism by which a diverse chemical space, as that conjectured to exist in a primordial Earth, might have lead to homochirality in living systems today.

\section{CONCLUSION}

Altogether, we have shown that a complex chemical network composed of chiral and achiral elements and sub- ject to random environmental drives can induce spontaneous breaking of chiral symmetry from totally symmetric starting conditions. This process was found to be strongly dependent on the dissipated-work difference between the chiral-breaking and racemic system, and is over-represented in systems achieving high dissipating states. Furthermore, strong, system-wide correlated driving was required to induce chiral-breaking states as otherwise strong but channel-independent correlations preserved the initial racemic states. Strikingly, these results demonstrate that chiral symmetry-breaking can be a purely energetically-driven process and stands in analogy to other continuity-breaking transitions like those of magnetic spin-models. They also imply that, experimentally, adaptable chemical systems might undergo spontaneous chiral symmetry breaking by virtue of a precise energy driving program and without explicit starting biases or autocatalytic constraints. These ideas invite us to expand the realm of possibilities in asymmetrical chemical synthesis, and pose an intriguing mechanism for the emergence of homochirality in a primordial chemical environment.

\section{METHODS}

In this work, we adapt a chemical framework introduced by Horowitz and England [20] and apply it to a chiral chemical system as follows. We consider a wellstirred solution of $N$ chemical elements inside a container of volume $V=1$ and kept at inverse temperature $\beta=1 / k_{\mathrm{B}} T=1$. We then construct a random network of $M$ chemical reactions of the form

$$
\sum_{i} a_{i}^{\alpha} X_{i} \rightleftharpoons \sum_{j} b_{j}^{\alpha} X_{j}, \quad \alpha=1, \ldots, M
$$

where the $a(b)$ coefficients are either 0 or 1 and $X_{i} \in$ $\left\{A_{i}, R_{j}, S_{k}\right\}$ with $N_{a}$ achiral $A_{i}$, and $N_{c}$ chiral $R_{j} / S_{j}$ elements. Most such reactions are unimolecular, but a fraction may also be bimolecular with probability $p_{b}$. Additionally, catalysts may be added with probability $p_{c}=0.5$ in the form of an independent element $X_{k}$ insertion on both sides of the reaction. Here we assume enantiomers to be mutually inert and to be perfectly enantioselective with respect to cross-species reactions. This is formally equivalent to considering separate $R$ and $S$ element sets and excluding any $R_{j} / S_{k}$ reaction pairings.

The rate constants $k_{\alpha}^{ \pm}$follow mass action kinetics,

$$
k_{\alpha}^{+}=k_{\alpha} \prod_{i} X_{i}^{a_{i}^{\alpha}}, \quad k_{\alpha}^{-}=k_{\alpha} \prod_{j} X_{j}^{b_{i}^{\alpha}}
$$

where $k_{\alpha}$ are random base rates chosen uniformly from the set of values $\left\{10^{-3}, 10^{-2}, 10^{-1}, 1\right\}$. A fraction of equilibrium reactions $\eta=M_{\text {driven }} / M$ are then duplicated, and rates altered by the addition of an environment force 
as $K_{\alpha}^{ \pm}=k_{\alpha}^{ \pm} e^{ \pm \beta F^{\gamma} / 2}$. This force form was chosen such that extremal function values from element configurations are rare[20, 24], and is given as

$$
F^{\gamma}(X)=\sum_{i>j} J_{i j}^{\gamma}\left(X_{i}-c_{i}^{\gamma}\right)\left(X_{j}-c_{j}^{\gamma}\right), \quad \gamma=1, \ldots, f
$$

where $J_{i j}$ are coupling strengths, of which only a fraction $\mu$ is non-zero, and chosen randomly from a uniform range between $[-s, s]$. The $c_{i}^{\gamma}$ are random offsets which take a value of either 0 or 1 . Driven reactions are then duplicated and mirrored via an argument swap in the reaction elements (if any) and forces, e.g., $F^{\gamma}\left(\ldots A_{i} R_{j} \ldots\right) \rightarrow F^{\gamma}\left(\ldots A_{i} S_{j} \ldots\right)$. This procedure ensures symmetrical driving in the system.

Finally, system noises are introduced in achiral-tochiral reactions $A_{i} \rightleftharpoons R_{i}\left(S_{i}\right)$ which add (subtract) a fraction $\delta_{i}$ from $R_{i}\left(S_{i}\right)$. Noises from the equivalent bimolecular reactions are omitted for simplicity and do not alter the nature of the results. These fluctuations are modeled as independent white noises for each reaction and chosen randomly from a uniform range $[0, \Delta]$, where $\Delta \ll 1$ represents maximum noise strength. In general, we found chiral-breaking models to arise generically for a wide-range of parameters and generally follow the original constraints necessary for high-dissipating systems [20]. Furthermore, we found noise history only altered the onset time of bifurcation but not the steady state in most models, with larger noise leading to shorter onset times. As a result, here we chose a parameter set that increased yield of chiral-breaking models per every round of 100 random generated models. For instance, systems with larger proportion of achiral elements yielded lower number of chiral-breaking models due to the larger number of ways mass can transfer between channels and thereby maintain a racemic state. Final parameters are thus as follows: $N_{a}=2, N_{c}=2 \times 12$, $p_{b}=0.3, \mu=0.25, f=10, s=0.2, \eta=0.6$, and $\Delta=0.02$.

The complete system dynamics follow standard mass action formulation but with additional noise terms. These noises are considered inherent to a reaction and not replicated for driven reactions. The overall chemical kinetics equations are then

$$
\begin{aligned}
\dot{\mathbf{X}} & =\left(\mathbf{b}_{\text {eq }}-\mathbf{a}_{\mathrm{eq}}\right)\left(\mathbf{k}_{\text {eq }}^{+}-\mathbf{k}_{\text {eq }}^{-}\right) \cdot(\mathbf{1}+\boldsymbol{\delta}) \\
& +\left(\mathbf{b}_{\text {driv }}-\mathbf{a}_{\text {driv }}\right)\left(\mathbf{K}_{\text {driv }}^{+}-\mathbf{K}_{\text {driv }}^{-}\right)
\end{aligned}
$$

where $\mathbf{k}_{\text {eq }}^{ \pm}$and $\mathbf{K}_{\text {driv }}^{ \pm}$represent column vectors of the equilibrium, and driven reaction rates respectively, and $\mathbf{b}-\mathbf{a}$ constitutes the corresponding stoichiometric matrix. The dot operator is the usual vector product with $\boldsymbol{\delta}$ representing system noises and $\mathbf{1}$ a unit vector. The stochastic differential equations are integrated using an Ito formulation and solved in Mathematica 12 with the ItoProcess method under an Euler-Maruyama scheme. To maintain accuracy a time step of $d t=5 \times 10^{-3}$ was used throughout. We found runtimes of $3 \times 10^{4}$ steps reached steady-state for most models.

\section{ACKNOWLEDGMENTS}

This work was publicly funded through the Institute for Basic Science, South Korea, Project Code IBS-R020D1.
[1] Y. Saito and H. Hyuga, Colloquium: Homochirality: Symmetry breaking in systems driven far from equilibrium, Rev. Mod. Phys. 85, 603 (2013).

[2] D. G. Blackmond, The origin of biological homochirality, Cold Spring Harbor Perspectives in Biology 2, 10.1101/cshperspect.a002147 (2010).

[3] R. M. Hazen, T. R. Filley, and G. A. Goodfriend, Selective adsorption of l- and d-amino acids on calcite: Implications for biochemical homochirality, Proceedings of the National Academy of Sciences 98, 5487 (2001).

[4] I. Weissbuch and M. Lahav, Crystalline architectures as templates of relevance to the origins of homochirality, Chemical Reviews 111, 3236 (2011).

[5] J. Bailey, A. Chrysostomou, J. H. Hough, T. M. Gledhill, A. McCall, S. Clark, F. Ménard, and M. Tamura, Circular polarization in star- formation regions: Implications for biomolecular homochirality, Science 281, 672 (1998).

[6] K. Soai, T. Kawasaki, and A. Matsumoto, Asymmetric autocatalysis of pyrimidyl alkanol and its application to the study on the origin of homochirality, Accounts of
Chemical Research 47, 3643 (2014).

[7] R. Plasson, D. K. Kondepudi, H. Bersini, A. Commeyras, and K. Asakura, Emergence of homochirality in far-fromequilibrium systems: Mechanisms and role in prebiotic chemistry, Chirality 19, 589 (2007).

[8] D. G. Blackmond, Autocatalytic models for the origin of biological homochirality, Chemical Reviews 120, 4831 (2020).

[9] J. M. Ribó, D. Hochberg, J. Crusats, Z. El-Hachemi, and A. Moyano, Spontaneous mirror symmetry breaking and origin of biological homochirality, Journal of The Royal Society Interface 14, 20170699 (2017).

[10] F. Frank, On spontaneous asymmetric synthesis, Biochimica et Biophysica Acta 11, 459 (1953).

[11] F. Jafarpour, T. Biancalani, and N. Goldenfeld, Noiseinduced mechanism for biological homochirality of early life self-replicators, Phys. Rev. Lett. 115, 158101 (2015).

[12] F. Jafarpour, T. Biancalani, and N. Goldenfeld, Noiseinduced symmetry breaking far from equilibrium and the emergence of biological homochirality, Phys. Rev. E 95, 
032407 (2017).

[13] G. Laurent, D. Lacoste, and P. Gaspard, Emergence of homochirality in large molecular systems, Proceedings of the National Academy of Sciences 118, 10.1073/pnas.2012741118 (2021).

[14] J. L. Baumgarten, Ferrochirality: A simple theoretical model of interacting dynamically invertible helical polymers, 1. the basic effects, Macromolecular Rapid Communications 15, 175 (1994).

[15] U. Hananel, A. Ben-Moshe, H. Diamant, and G. Markovich, Spontaneous and directed symmetry breaking in the formation of chiral nanocrystals, Proceedings of the National Academy of Sciences 116, 11159 (2019).

[16] R. E. Breier, R. L. B. Selinger, G. Ciccotti, S. Herminghaus, and M. G. Mazza, Spontaneous chiral symmetry breaking in collective active motion, Phys. Rev. E 93, 022410 (2016).

[17] R. Plasson, H. Bersini, and A. Commeyras, Recycling frank: Spontaneous emergence of homochirality in noncatalytic systems, Proceedings of the National Academy of Sciences 101, 16733 (2004).

[18] R. Plasson and H. Bersini, Energetic and entropic anal- ysis of mirror symmetry breaking processes in a recycled microreversible chemical system, The Journal of Physical Chemistry B 113, 3477 (2009).

[19] S. I. Walker, Origins of life: a problem for physics, a key issues review, Reports on Progress in Physics 80, 092601 (2017).

[20] J. M. Horowitz and J. L. England, Spontaneous finetuning to environment in many-species chemical reaction networks, Proceedings of the National Academy of Sciences 114, 7565 (2017).

[21] T. Kachman, J. A. Owen, and J. L. England, Selforganized resonance during search of a diverse chemical space, Phys. Rev. Lett. 119, 038001 (2017).

[22] D. Kondepudi, B. Kay, and J. Dixon, End-directed evolution and the emergence of energy-seeking behavior in a complex system, Phys. Rev. E 91, 050902 (2015).

[23] E. te Brinke, J. Groen, A. Herrmann, H. A. Heus, G. Rivas, E. Spruijt, and W. T. S. Huck, Dissipative adaptation in driven self-assembly leading to self-dividing fibrils, Nature Nanotechnology 13, 849 (2018).

[24] D. Sherrington and S. Kirkpatrick, Solvable model of a spin-glass, Phys. Rev. Lett. 35, 1792 (1975). 


\section{Supplementary Material}

\section{ILLUSTRATION OF RARE OSCILLATORY SOLUTIONS}
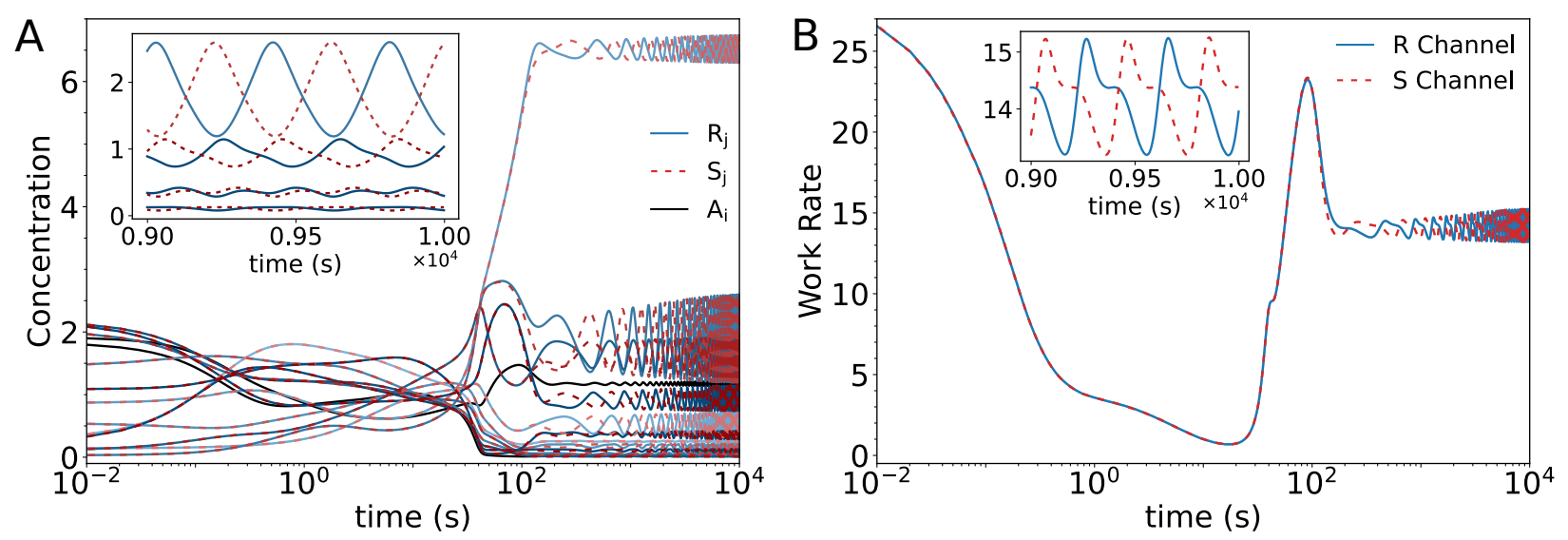

FIG. S1. An example of an oscillating system with oscillating chiral element oscillations but average $e e=0$. Left shows element concentration profiles as a function of time and right the corresponding work rate plots. Insets depict the last 1,000 steps for selected elements (left) and workrates (right) showing clear oscillatory behavior. 


\section{ILLUSTRATION OF SOLUTION DISTRIBUTIONS FOR DIFFERENT PA- RAMETER SETS}
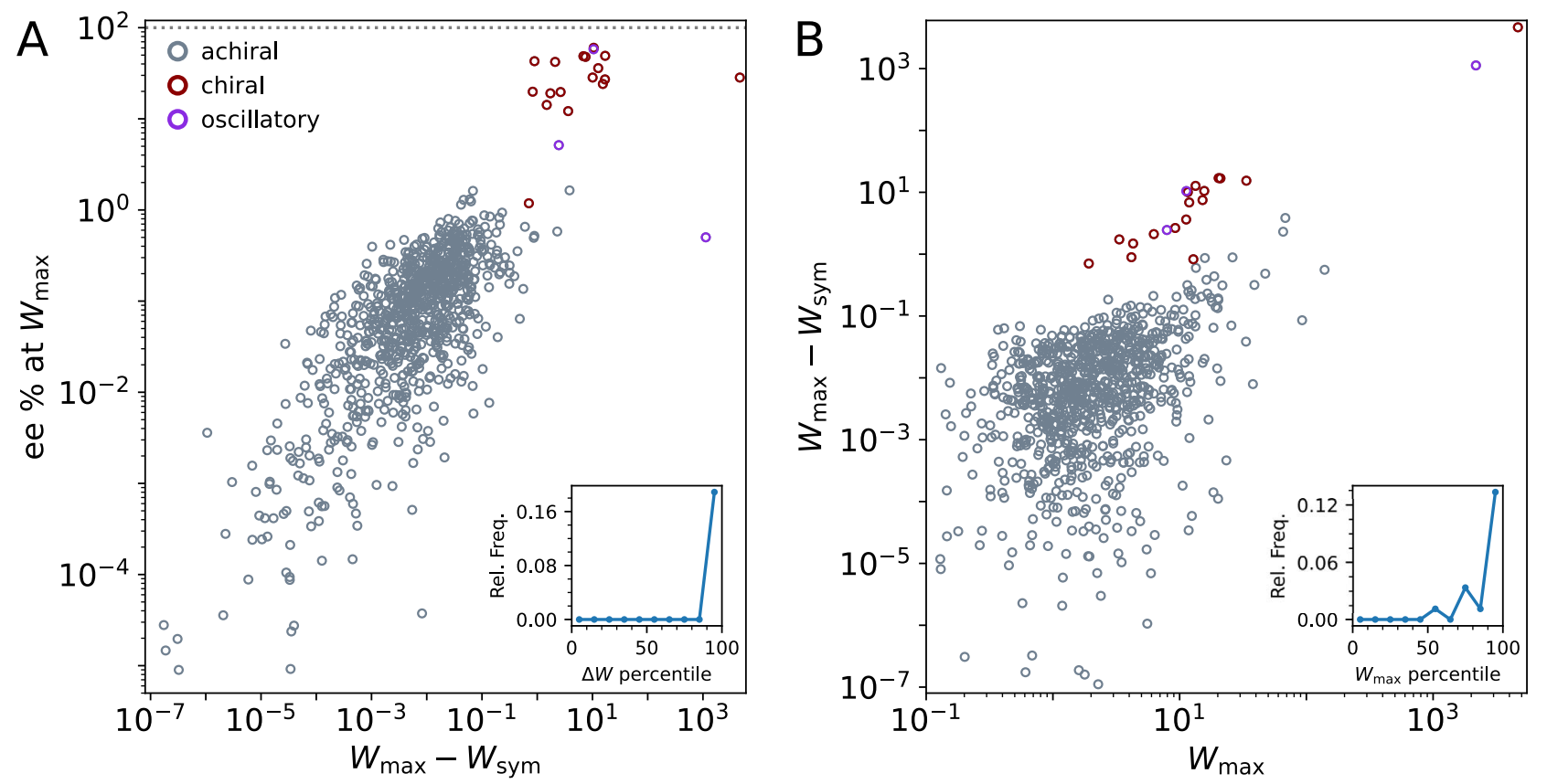

FIG. S2. A. Plot of enantiomeric excess ee vs. $\Delta W=W_{\max }-W_{\text {sym }}$ and B. $\Delta W$ vs $W_{\max }$ for 900 distinct models from three different parameter sets (300 each). Points below $\sim 10^{-7}$ values cut-off for clarity. Insets: A. relative population frequency of chiral-breaking models as a function of the work difference percentile and $\mathrm{B}$. the maximum work $W_{\max }$. Parameter sets are given as i) $N_{a}=2, N_{c}=2 \times 12, \mu=0.3, s=0.3, \eta=0.3$; ii) $N_{a}=8, N_{c}=2 \times 9, \mu=0.25, s=0.2, \eta=0.6$; and iii) $N_{a}=8, N_{c}=2 \times 9, \mu=0.3, s=0.3, \eta=0.3$, with all sets $p_{c}=0.5, p_{b}=0.3, f=10$ and $\Delta=0.02$. 